\title{
NASA faces legal challenge over Hawaiian telescope plan
}

Tony Reichhardt, Washington

A Hawaiian state agency is suing NASA over plans to upgrade the twin Keck telescopes on the mountain of Mauna Kea - the premier site for astronomical observation in the Northern Hemisphere.

The lawsuit, which was filed last week by the state's Office of Hawaiian Affairs, demands that the space agency completes a full Environmental Impact Statement (EIS) before carrying out its plan to build four to six small 'outrigger' telescopes that would improve Keck's ability to reveal planetary systems around distant stars. The case is seen as a significant escalation in a longrunning battle between astronomers and native cultural groups in Hawaii.

NASA had hoped to be granted a state permit this month to begin construction later this year, and aimed to begin operating the outriggers in 2003. But completing an EIS would probably delay the project by a year or more, adding millions to its $\$ 50$ million price tag.

The outriggers would operate as an interferometer with the two existing 10-metre Keck telescopes. This effectively produces the resolution of a much larger instrument. NASA wants to upgrade the facility primarily to study planets outside our Solar System, and says that four of its six science objectives cannot be accomplished without the outriggers.

The agency had hoped that a less formal "environmental assessment" of the outriggers' impact on the mountain would satisfy native
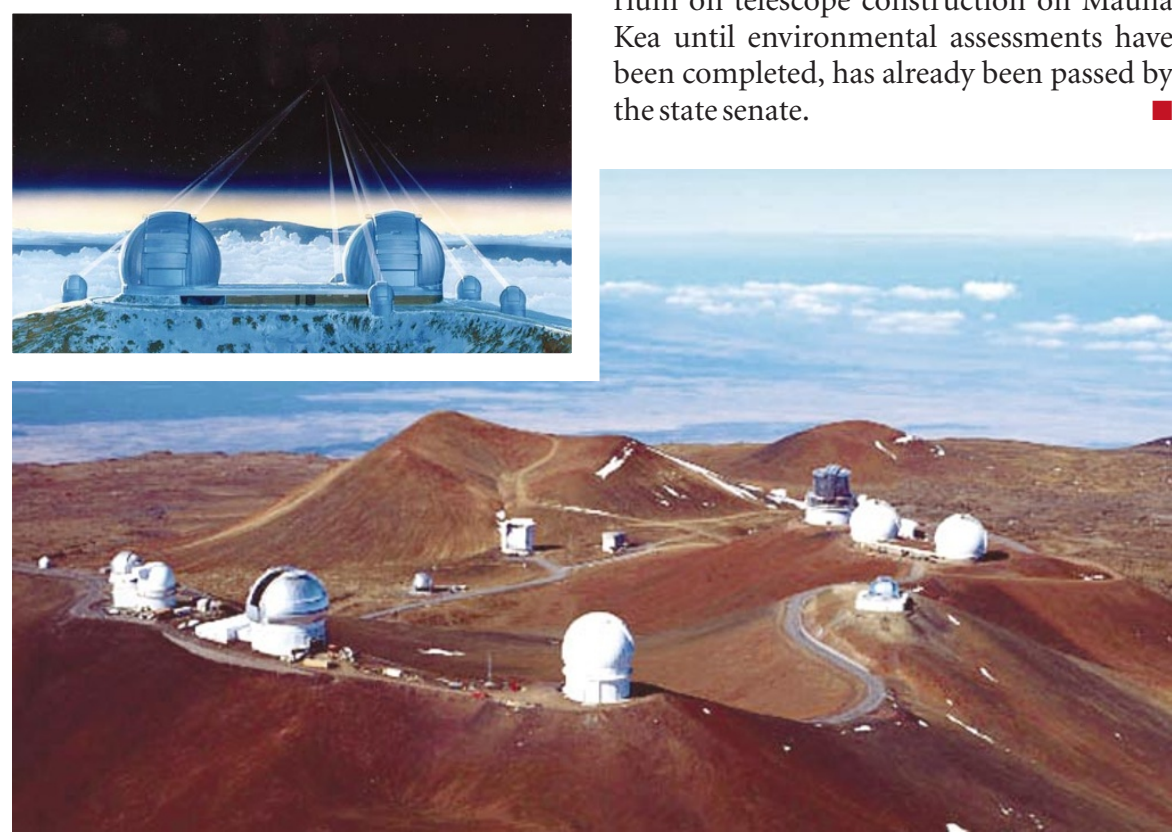

NASA's proposed upgrade (inset) of Keck has provoked opposition from native Hawaiian groups.

Hawaiian groups, who have objected to the building of telescopes on a site that they hold sacred (see Nature 410, 1015; 2001). In February, NASA proposed having cultural and archaeological monitors present during construction, and offered \$2 million in funding for cultural preservation and education projects in Hawaii. It also outlined a mitigation plan for the Wekiu bug, a threatened insect species that lives on Mauna Kea.

But the controversy "goes beyond the physical details to the spiritual aspects", says Bill Stormont, director of the Office of Mauna Kea Management, which was set up by the University of Hawaii to manage the science reserve on the mountain. And many astronomers fear that the NASA case is simply a symptom of a worsening relationship between themselves and native Hawaiian groups, which may ultimately threaten Mauna Kea's future as an observation site.

Although NASA's outriggers are the only significant construction planned on the mountain for the next several years, the University of California is eyeing the site for its California Extremely Large Telescope project. Mauna Kea is also considered a logical site for a possible Next Generation Large Telescope project.

But such plans are facing an increasing number of potential roadblocks. A bill introduced in the Hawaiian legislature in March, for example, called for astronomers to begin paying rent to use mountains. Although the proposal died during committee deliberations, another bill, which calls for a moratorium on telescope construction on Mauna Kea until environmental assessments have been completed, has already been passed by the state senate.

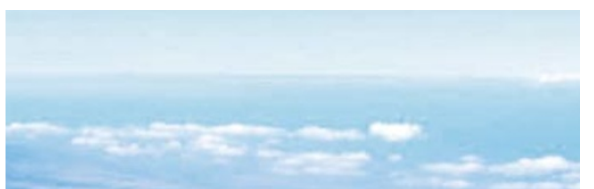

\section{Neutrino review will seek out 'scientific redundancy'}

Geoff Brumfiel, Washington

The White House has ordered a rapid-fire study of two proposed neutrino projects, raising the prospect of some tough choices ahead for the US physics community.

The projects to be reviewed are the IceCube neutrino observatory and the National Underground Science Laboratory (NUSL). Each would cost hundreds of millions of dollars to build, and both are devoted to the detection of neutrinos tiny, almost massless particles that are of intense interest to physicists.

In a 29 March letter, John Marburger, director of the White House Office of Science and Technology Policy, asked the National Academy of Sciences for a study of the respective merits and of "any possible scientific redundancy" between the projects, to be completed by September.

IceCube is an array of light-sensitive detectors buried within a cubic kilometre of Antarctic ice. The detectors are designed to watch for collisions between the ice molecules and high-energy neutrinos from deep space. From these collisions, astronomers hope to trace the neutrinos back to their extragalactic origins.

The international project, led by the National Science Foundation (NSF), has undergone several reviews - according its leader, Francis Halzen of the University of Wisconsin at Madison, "they will have a hard time finding a person who has not reviewed it already".

The NUSL is new by comparison. Plans for its construction were submitted to the NSF only last June, and have yet to be endorsed by its governing National Science Board. But NUSL is riding a strong wave of scientific and political support (see Nature 416,775 ; 2002).

The underground laboratory would detect low-energy neutrinos and seek to determine their properties, as well as providing a home for proton-decay experiments, searches for 'dark matter' and geomicrobiology. "There is virtually no overlap in the physics of these two projects," says Wick Haxton of the University of Washington, who heads the NUSL plan.

But the NSF has a limited budget for major facilities, and budget hawks at the White House Office of Management and Budget, whose interest is said to have led to the study request, want to keep it that way. Project advocates hope the study will convince them that the two projects do not overlap. 